\title{
ON THE PERTURBATION OF SINGULAR ANALYTIC MATRIX FUNCTIONS: A GENERALIZATION OF LANGER AND NAJMAN'S RESULTS
}

\author{
FERNANDo De TERÁN
}

Abstract. Given a singular $n \times n$ matrix function $A(\lambda)$, analytic in a neighborhood of an eigenvalue $\lambda_{0} \in \mathbb{C}$, and perturbations, $B(\lambda, \varepsilon)$, such that $B(\lambda, 0) \equiv 0$ and analytic in $\lambda$ and $\varepsilon$ near $\left(\lambda_{0}, 0\right)$, we provide sufficient conditions on these perturbations for the existence of eigenvalue expansions of the perturbed matrix $A(\lambda)+B(\lambda, \varepsilon)$ near $\lambda_{0}$. We also describe the first order term of these expansions. This extends to the singular case some results by Langer and Najman.

Mathematics subject classification (2010): 15A18, 47A56.

Keywords and phrases: Singular analytic matrix function, eigenvalue problem, perturbation, Puiseux expansions.

\section{REFERENCES}

[1] J. A. Ball, I. Gohberg and L. Rodman, Interpolation of Rational Matrix Functions, Oper. Theory Adv. Appl. 45, Birkhäuser, Basel, 1990.

[2] F. DE TERÁN AND F. M. DOPICO, First order spectral perturbation theory of square singular matrix polynomials, Linear Algebra Appl., 432 (2010), 892-910.

[3] F. De Terán, F. M. Dopico And J. Moro, First order spectral perturbation theory of square singular matrix pencils, Linear Algebra Appl., 429 (2008), 548-576.

[4] P. Lancaster, A. S. Markus And F. Zhou, Perturbation theory for analytic matrix functions: the semisimple case, SIAM J. Matrix Anal. Appl., 25 (2003), 606-626.

[5] H. LANGer AND B. NAJMAn, Remarks on the perturbation of analytic matrix functions II, Integr. Equat. Oper. Th., 12 (1989), 392-407.

[6] H. LANGer AND B. NAJMAn, Remarks on the perturbation of analytic matrix functions III, Integr. Equat. Oper. Th., 15 (1992), 796-806.

[7] J. G. Sun, Orthogonal projections and the perturbation of the eigenvalues of singular pencils, J. Comp. Math., 1 (1983), 63-74.

[8] M. M. Vainberg And V. A. Trenogin, The methods of Lyapunov and Schmidt in the Theory of Non-Linear Equations and their further development, P. Noordhoff, Leyden, 1974.

[9] P. VAn Dooren, Reducing subspaces: Definitions, properties, and algorithms, in: B. Kågström and A. Ruhe (Eds.), Matrix Pencils, Lecture Notes in Mathematics, Vol. 973, Springer-Verlag, Berlin, 1983 , pp. 58-73. 